\title{
A point group approach to selection rules in crystals
}

\author{
(C) V.P. Smirnov, R.A. Evarestov*, P. Tronc** \\ Institute of Fine Mechanics and Optics, \\ 197101 St. Petersburg, Russia \\ * St. Petersburg State University, \\ 198904 St. Petersburg, Russia \\ ** Ecole Supérieure de Physique et Chimie Industrielles, \\ F-75005 Paris, France
}

(Submitted 23 January 2003)

\begin{abstract}
The problem of generation of the selection rules for a transition between Bloch states at any point of the Brillouin zone in crystals is equivalent to the problem of the decomposition of Kronecker products of two representations (reps) of a space group into irreducible components (the full group method). This problem can be solved also by the subgroup method where small reps of little groups are used. In this article, we propose the third method of the selection rules' generation which is formulated in terms of projective reps of crystal point groups. It is based on a well known relation between small irreducible reps (irreps) of little space groups and projective irreps of corresponding little co-groups. The proposed procedure is illustrated by calculations of the Kronecker products for different irreps at the $W$ point of the Brillouin zone for the nonsymmorphic space group $O_{h}^{7}$ being one of the most complicated space groups for the selection rules' generation. As an example, the general procedure suggested is applied to obtain the selection rules for direct and phonon-assisted electrical dipole transitions between some states in crystals with the space group $O_{h}^{7}$.
\end{abstract}

One of the authors (V.P.S.) acknowledges the support of Ministére de la Recherche (France).

\section{Introduction}

The knowledge of selection rules is well known to be of a great importance in the study of optical properties of crystals, electron-phonon interaction and phase transitions in solids. It is evident that the generation of selection rules for transitions between states related to the center $\mathbf{k}=0$ of the Brillouin zone (BZ) can be expressed in terms of representations (reps) of the crystal point group. For direct transitions between the states with $\mathbf{k} \neq 0$ and for indirect transitions, the generation of selection rules is more complex because of complicated structure of space group reps (subgroup method [1-4], full group method [5]).

The optical properties at the $\Gamma$ point in the BZ of materials used for optoelectronic devices are of crucial importance since the materials are direct gap semiconductors. Among them, one can find stoichiometric crystals (such as GaAs, InP or $\mathrm{CaN}$ ) and alloys (for example, ternary compounds like AlGaAs or quaternary ones such as GaInAsS and $\mathrm{GaInAsSb}$ ). In any of these materials, the fundamental optical transition takes place at the $\Gamma$ point insuring strong absorption and recombination. By varying the ratios of various elements in the alloys, it is possible to tailor the band gap value at $\Gamma$ point to fit the required operating wavelength. Many nanostructures such as quantum wells (QW's), superlattices (SL's), quantum wires (QWI's) and quantum dots (QD's) are made of the materials mentioned above. Nanostructures are usually studied in the envelope function approximation based on the properties of the Bloch functions at the $\Gamma$ point in the BZ of the bulk materials they are built from.

On the other hand, germanium Ge and silicon $\mathrm{Si}$ (the most widely used semiconductors) have indirect gaps.
Silicon is used to built integrated circuits and other devices based on charge transport phenomena (GaAs and related compounds can also be used for such devices when their high carrier mobility is needed). Some nanostructures, such as type II GaAs/AlAs SL's have also indirect gaps.

In any bulk semiconductor or semiconductor structure, it is necessary to study direct transitions also at points in the BZ other than $\Gamma$ and indirect transitions when one or both states correspond to $\mathbf{k} \neq 0$ (participation of a particle with a finite wave vector). The more frequent case is that of phonon assisted transitions. Note that the high symmetry points in the BZ are particularly important since they generally correspond to high density of phonon states. Selection rules' deduction in general case is much more complicated since the symmetry of the Bloch states and hence the selection rules depend of the location of the BZ points involved in the process.

In Chapter 4 of Ref. [3], the procedure of generation of the selection rules in crystals is based on a sufficiently refined mathematical groundwork (double and triple coset decompositions of space groups, use of the Mackey theorem for induced reps). This procedure was realized in the computer program, the results being collected in the threevolume Tables [4].

The more simple approach to the selection rules' problem may be developed basing on the well-known relation between small irreducible reps (irreps) of little space groups and projective irreps of corresponding little co-groups [6,7]. We demonstrate in this paper that the procedure of generation of the selection rules for a transition between any states in crystals can be formulated in terms of projective irreps of point groups. 
In Section 2, all the necessary notations are introduced and the connection between irreps of space groups and projective irreps of point groups is considered in detail. The general procedure of generation of the selection rules is formulated in Section 3. Each step of its realization is illustrated by calculations of the Kronecker products for different irreps at the $W$ point of the BZ for nonsymmorphic space group $O_{h}^{7}$ which is one of the most complicated space groups for the selection rules' generation. In Section 4, as an example, the general procedure is applied to obtain the selection rules for direct and phonon-assisted electrical dipole transitions between some states in crystals with space group $O_{h}^{7}$.

\section{Connection between small representations of space groups and projective representations of point groups}

Let the space group $G$ of a crystal consist of the elements $g=\left(R \mid \mathbf{v}_{R}+\mathbf{a}_{\mathbf{n}}\right) \in G$ where the orthogonal operation $R$ is followed by the improper translation $\mathbf{v}_{R}$ and lattice translation $\mathbf{a}_{\mathbf{n}}$. The vectors $\mathbf{a}_{\mathbf{n}}$ form the invariant subgroup $T$ of the space group $G(T \triangleleft G)$. The point group $F$ of the $n_{F}$ orthogonal operations $R$ describes the symmetry of directions in the crystal and is called crystalline class or point symmetry group of the crystal. The set of left cosets $\left(R_{i} \mid \mathbf{v}_{i}\right) T$ in the decomposition of $G$ with respect to the translation subgroup $T$

$$
G=\sum_{i=1}^{n_{F}}\left(R_{i} \mid \mathbf{v}_{i}\right) T
$$

forms a factor group $G / T$ isomorphic to the point group $F$ $(F \leftrightarrow G / T)$ of order $n_{F}$.

The translation group $T$ is Abelian. All its irreps are onedimensional and are classified by wave vectors $\mathbf{k}$ in the $\mathrm{BZ}$

$$
d^{(\mathbf{k})}\left(\mathbf{a}_{\mathbf{n}}\right)=\exp \left(-i \mathbf{k} \mathbf{a}_{\mathbf{n}}\right) .
$$

The elements $g \in G$ leaving the wave vector $\mathbf{k}$ invariant up to reciprocal lattice vector $\mathbf{B}_{\mathbf{m}}$

$$
g^{(\mathbf{k})} \mathbf{k}=R^{(\mathbf{k})} \mathbf{k}=\mathbf{k}+\mathbf{B}_{\mathbf{m}}
$$

form the little group $G_{\mathbf{k}}$ of the wave vector $\mathbf{k}$. The group $G_{-\mathbf{k}}$ consists of the same elements as the group $G_{\mathbf{k}}$. The little co-group $F_{\mathbf{k}}=F_{-\mathbf{k}}$ includes the elements $R^{(\mathbf{k})}$ $\left(\left(R^{(\mathbf{k})} \mid \mathbf{v}_{R^{(\mathbf{k})}}\right) \in G_{\mathbf{k}}\right)$. The representatives $g_{i}=\left(R_{j} \mid \mathbf{v}_{j}\right)$ of left cosets $g_{j} G_{\mathbf{k}}$ in the decomposition of $G$ with respect to $G_{\mathbf{k}} \subset G$

$$
G=\sum_{j=1}^{t} g_{j} G_{\mathbf{k}} \quad g_{1}=(E \mid 0)
$$

determine the so-called irreducible star ${ }^{*} \mathbf{k}$ of the wave vector $\mathbf{k}$ consisting of $t$ wave vectors

$$
{ }^{*} \mathbf{k}: \quad \mathbf{k}_{j}=g_{j} \mathbf{k}=R_{j} \mathbf{k}, \quad j=1,2, \ldots, t .
$$

The little groups $G_{\mathbf{k}_{j}}$ for different points of the star ${ }^{*} \mathbf{k}$ are isomorphous to the little group $G_{\mathbf{k}}$

$$
G_{\mathbf{k}_{j}}=g_{j} G_{\mathbf{k}} g_{j}^{-1} \text {. }
$$

The irreps of $G$ (full irreps) are labeled by the irreducible star ${ }^{*} \mathbf{k}$ of the wave vector $\mathbf{k}$ and by the index $\gamma$ numbering the inequivalent irreps within the same star $\left.{ }^{*} \mathbf{k}:[G]_{\gamma}{ }^{*} \mathbf{k}\right)$. The full irrep $[G]_{\gamma}^{\left({ }^{*} \mathbf{k}\right)}$ of $G$ is in a one-to-one correspondence with the small irrep $\left[G_{\mathbf{k}}\right]_{\gamma}$ of $G_{\mathbf{k}} \subset G$ and is obtained from the latter by induction procedure $[7,8]$

$$
[G]_{\gamma}^{(* \mathbf{k})}=\left[G_{\mathbf{k}}\right]_{\gamma} \uparrow G .
$$

The set of all small irreps of all little groups $G_{\mathbf{k}}$ with $\mathbf{k}$ being in a representation domain of the BZ determines unambiguously all the irreps of the space group $G$. That is why the Tables of space group irreps, as a rule, contain the small irreps of little groups $G_{\mathbf{k}}[3,9-12]$.

The matrices $D^{\left(\left[G_{\mathbf{k}}\right]_{\gamma}\right)}\left(g_{i, \mathbf{n}}\right)\left(g_{i, \mathbf{n}} \in G_{\mathbf{k}}\right)$ of the small irreps $\left[G_{\mathbf{k}}\right]_{\gamma}$ of $G_{\mathbf{k}}$ are in one-to-one correspondence with the matrices $d^{\left(\left[F_{\mathbf{k}}\right]_{\gamma}\right)}\left(R_{i}\right)$ of so-called projective irreps $\left[F_{\mathbf{k}}\right]_{\gamma}$ of $F_{\mathbf{k}}$ as follows

$$
\begin{gathered}
D^{\left(\left[G_{\mathbf{k}}\right]_{\gamma}\right)}\left(g_{i, \mathbf{n}}\right)=e^{-i \mathbf{k}_{\mathbf{n}}} d^{\left(\left[F_{\mathbf{k}}\right]_{\gamma}\right)}\left(R_{i}\right), \\
g_{i, \mathbf{n}}=\left(R_{i} \mid \mathbf{v}_{i}+\mathbf{a}_{\mathbf{n}}\right) \in G_{\mathbf{k}}, \quad R_{i} \in F_{\mathbf{k}} .
\end{gathered}
$$

In particular, the matrices $D^{\left(\left[G_{\mathbf{k}}\right]_{\gamma}\right)}\left(g_{i, 0}\right)$ and $d^{\left(\left[F_{\mathbf{k}}\right]_{\gamma}\right)}\left(R_{i}\right)$ coincide

$$
\begin{gathered}
D^{\left(\left[G_{\mathbf{k}}\right]_{\gamma}\right)}\left(g_{i, 0}\right)=d^{\left(\left[F_{\mathbf{k}}\right]_{\gamma}\right)}\left(R_{i}\right), \\
g_{i, 0}=\left(R_{i} \mid \mathbf{v}_{i}\right) \in G_{\mathbf{k}}, \quad R_{i} \in F_{\mathbf{k}} .
\end{gathered}
$$

The multiplication law for the matrices $d^{\left(\left[F_{\mathrm{k}}\right]_{\gamma}\right)}\left(R_{i}\right)$ of projective irreps $\left[F_{\mathbf{k}}\right]_{\gamma}$ of a co-group $F_{\mathbf{k}}$ follows from the multiplication law for space group elements

$$
d^{\left(\left[F_{\mathbf{k}}\right]_{\gamma}\right)}\left(R_{i}\right) d^{\left(\left[F_{\mathbf{k}}\right]_{\gamma}\right)}\left(R_{i^{\prime}}\right)=d^{\left(\left[F_{\mathbf{k}}\right]_{\gamma}\right)}\left(R_{i} R_{i^{\prime}}\right) \omega^{(\mathbf{k})}\left(R_{i}, R_{i^{\prime}}\right),
$$

where the set of

$$
\begin{aligned}
& \omega^{(\mathbf{k})}\left(R_{i}, R_{i^{\prime}}\right)=e^{-i \mathbf{k}\left(\mathbf{v}_{i}+R_{i} \mathbf{v}_{i^{\prime}}-\mathbf{v}_{i, i^{\prime}}\right)}, \\
& \left|\omega\left(R_{i}, R_{i^{\prime}}\right)\right|^{2}=1, \quad R_{i}, R_{i^{\prime}} \in F_{\mathbf{k}}
\end{aligned}
$$

is a factor system for the projective irreps $\left[F_{\mathbf{k}}\right]_{\gamma}$ $\left(g_{i i^{\prime}, 0}=\left(R_{i}, R_{i^{\prime}} \mid \mathbf{v}_{i i^{\prime}}\right) \in G_{\mathbf{k}}\right)$. The characters of these projective irreps can be taken directly from Tables $[3,9,10,12]$. If all the factors (11) are equal to unit, the projective irrep becomes an ordinary one. In particular, this is the case of all the little co-groups $F_{\mathbf{k}}$ of all the symmorphic space groups (since all $\mathbf{v}_{j^{\prime}}=0$ ).

There exist projective irreps $\overline{\left[F_{\mathbf{k}}\right]_{y}}$, with another choice of the factor system ( $p$-equivalent to $\left[F_{\mathbf{k}}\right]_{\gamma}$ )

$$
\begin{gathered}
D^{\left(\left[F_{\mathbf{k}}\right]_{\gamma}\right)}\left(g_{i, \mathbf{n}}\right)=e^{-i \mathbf{k}\left(\mathbf{v}_{i}+\mathbf{a}_{\mathbf{n}}\right)} d^{\left(\overline{\left[F_{\mathbf{k}}\right]_{y}}\right)}\left(R_{i}\right), \\
g_{i, \mathbf{n}}=\left(R_{i} \mid \mathbf{v}_{i}+\mathbf{a}_{\mathbf{n}}\right) \in G_{\mathbf{k}}, \quad R_{i} \in F_{\mathbf{k}}, \\
\bar{\omega}^{(\mathbf{k})}\left(R_{i}, R_{i^{\prime}}\right)=e^{i\left(\mathbf{k}-R_{i}^{-1} \mathbf{k}\right) \mathbf{v}_{i^{\prime}}}, \\
\left|\bar{\omega}\left(R_{i}, R_{i^{\prime}}\right)\right|^{2}=1, \quad R_{i}, R_{i^{\prime}} \in F_{\mathbf{k}} .
\end{gathered}
$$


They are used in Ref. [11]. The matrices $D^{\left(\left[G_{\mathbf{k}}\right]_{\gamma}\right)}\left(g_{i, 0}\right)$ and $d^{\left.\left(\overline{F_{\mathbf{k}}}\right]_{\nu}\right)}\left(R_{i}\right)$ differ by the factor $e^{-i \mathbf{k v}_{i}}$ only

$$
\begin{aligned}
& D^{\left(\left[F_{\mathbf{k}}\right]_{\gamma}\right)}\left(g_{i, 0}\right)=e^{-i \mathbf{k} \mathbf{v}_{i}} d^{\left(\overline{\left[F_{\mathbf{k}}\right]_{\gamma}}\right)}\left(R_{i}\right), \\
& g_{i, 0}=\left(R_{i} \mid \mathbf{v}_{i}\right) \in G_{\mathbf{k}}, \quad R_{i} \in F_{\mathbf{k}} .
\end{aligned}
$$

Let relation (8) between the reps $\left[G_{\mathbf{k}}\right]_{\gamma}$ of $G_{\mathbf{k}}$ and $\left[F_{\mathbf{k}}\right]_{\gamma}$ of $F_{\mathbf{k}}$ be denoted by the symbols

$$
\left[G_{\mathbf{k}}\right]_{\gamma}=\left[F_{\mathbf{k}}\right]_{\gamma} \Uparrow G_{\mathbf{k}}, \quad\left[F_{\mathbf{k}}\right]_{\gamma}=\left[G_{\mathbf{k}}\right]_{\gamma} \Downarrow F_{\mathbf{k}} .
$$

Then the relation between the irreps $[G]_{\gamma}^{\left({ }^{*} \mathbf{k}\right)}$ of space group $G$ and the projective irreps $\left[F_{\mathbf{k}}\right]_{\gamma}$ or ${\overline{\left[F_{\mathbf{k}}\right.}}_{\gamma}$ of little co-groups has the form

$$
[G]_{\gamma}^{\left({ }^{*} \mathbf{k}\right)}=\left(\left[F_{\mathbf{k}}\right]_{\gamma} \Uparrow G_{\mathbf{k}}\right) \uparrow G, \text { or }[G]_{\gamma}^{\left({ }^{*} \mathbf{k}\right)}=\left({\overline{\left[F_{\mathbf{k}}\right.}}_{\gamma} \Uparrow G_{\mathbf{k}}\right) \uparrow G .
$$

The basis functions of irreps $[G]_{\gamma}^{(* \mathbf{k})}$ of a space group $G$ can be always chosen as being the basis functions of small irreps $\left[G_{\mathbf{k}}\right]_{\gamma}$ of little groups $G_{\mathbf{k}}$ and projective irreps $\left[F_{\mathbf{k}}\right]_{\gamma}$ (or $\overline{\left[F_{k}\right]_{\gamma}}$ ) of little co-groups $F_{\mathbf{k}}$.

For the selection rules' generation, it is necessary to consider the direct product of small reps of two of the little groups. The latter is possible only for the common elements of little groups, i.e. for their intersection. Let $\left[G_{\mathbf{k}_{1}}\right]_{\alpha}$ and $\left[G_{\mathbf{k}_{2}}\right]_{\beta}$ be small reps of two little groups $G_{\mathbf{k}_{1}}$ and $G_{\mathbf{k}_{2}}$. The direct product of their subductions on their intersection $\left(\left[G_{\mathbf{k}_{1}}\right]_{\alpha} \downarrow\left(G_{\mathbf{k}_{1}} \cap G_{\mathbf{k}_{2}}\right) \times\left[G_{\mathbf{k}_{2}}\right]_{\beta} \downarrow\left(G_{\mathbf{k}_{1}} \cap G_{\mathbf{k}_{2}}\right)\right)$ is a small rep of the group $\left(G_{\mathbf{k}_{1}} \cap G_{\mathbf{k}_{2}}\right)$. Every element $g_{i, 0} \in\left(G_{\mathbf{k}_{1}} \cap G_{\mathbf{k}_{2}}\right)$ leaves invariant the wave vectors $\mathbf{k}_{1}$ and $\mathbf{k}_{2}$ and, therefore, their sum $\mathbf{k}_{3}=\mathbf{k}_{1}+\mathbf{k}_{2}$ : $\left(G_{\mathbf{k}_{1}} \cap G_{\mathbf{k}_{2}}\right) \subseteq G_{\mathbf{k}_{3}}$. The little group $G_{\mathbf{k}_{3}}$ has no other common elements either with $G_{\mathbf{k}_{1}}$ or with $G_{\mathbf{k}_{2}}$. Indeed, let us assume the contrary that

$$
\tilde{g} \in G_{\mathbf{k}_{3}}, \quad \tilde{g} \in G_{\mathbf{k}_{1}}, \quad \tilde{g} \notin\left(G_{\mathbf{k}_{1}} \cap G_{\mathbf{k}_{2}}\right) .
$$

Such an element $\tilde{g}$ would leave invariant $\mathbf{k}_{3}$ and $\mathbf{k}_{1}$ and, therefore, $\mathbf{k}_{2}=\mathbf{k}_{3}-\mathbf{k}_{1}$, i. e. it would be contained in $G_{\mathbf{k}_{1}} \cap G_{\mathbf{k}_{2}}$ in contradiction with the initial assumption.

Let $d^{\left(\left[F_{\mathbf{k}_{1}}\right]_{\alpha}\right)}$ and $d^{\left(\left[F_{\mathbf{k}_{2}}\right]_{\beta}\right)}$ be the matrices of subductions of the projective reps $\left[F_{\mathbf{k}_{1}}\right]_{\alpha} \downarrow\left(F_{\mathbf{k}_{1}} \cap F_{\mathbf{k}_{2}}\right)$ and $\left[F_{\mathbf{k}_{2}}\right]_{\beta} \downarrow\left(F_{\mathbf{k}_{1}} \cap F_{\mathbf{k}_{2}}\right)$ of two little co-groups $F_{\mathbf{k}_{1}}$ and $F_{\mathbf{k}_{2}}$ with factor systems $\omega^{\left(\mathbf{k}_{1}\right)}\left(R_{i}, R_{j}\right)$ and $\omega^{\left(\mathbf{k}_{2}\right)}\left(R_{i}, R_{j}\right)$ $\left(R_{i}, R_{j} \in F_{\mathbf{k}_{1}} \cap F_{\mathbf{k}_{2}}\right)$, respectively. The direct product $d^{\left(\left[F_{\mathbf{k}_{1}}\right]_{\alpha}\right)} \times d^{\left(\left[F_{\mathbf{k}_{2}}\right]_{\beta}\right)}$ is a projective rep $d^{\left(\left[F_{\mathbf{k}_{3}}\right]_{\alpha \beta}\right)}\left(\mathbf{k}_{3}=\mathbf{k}_{1}+\mathbf{k}_{2}\right)$ of the group $\left(F_{\mathbf{k}_{1}} \cap F_{\mathbf{k}_{2}}\right) \subset F_{\mathbf{k}_{3}}$ with the factor system $\omega^{\left(\mathbf{k}_{3}\right)}\left(R_{i}, R_{j}\right)=\omega^{\left(\mathbf{k}_{1}\right)}\left(R_{i}, R_{j}\right) \omega^{\left(\mathbf{k}_{2}\right)}\left(R_{i}, R_{j}\right)$. Indeed, let

$$
\begin{gathered}
d^{\left(\left[F_{\mathbf{k}_{3}}\right]_{\alpha \beta}\right)}(R) \equiv d^{\left(\left[F_{\mathbf{k}_{1}}\right]_{\alpha}\right)}(R) \times d^{\left(\left[F_{\mathbf{k}_{2}}\right]_{\beta}\right)}(R), \\
R \in\left(F_{\mathbf{k}_{1}} \cap F_{\mathbf{k}_{2}}\right)
\end{gathered}
$$

be matrices of the direct product of two projective reps. Then

$$
\begin{aligned}
& d^{\left(\left[F_{\mathbf{k}_{3}}\right] \alpha \beta\right.}\left(R_{1}\right) \cdot d^{\left(\left[F_{\mathbf{k}_{3}}\right] \alpha \beta\right)}\left(R_{2}\right) \\
&=\left\{d^{\left(\left[F_{\mathbf{k}_{1}}\right] \alpha\right)}\left(R_{1}\right) \times d^{\left(\left[F_{\mathbf{k}_{2}}\right] \beta\right)}\left(R_{1}\right)\right\}\left\{d^{\left(\left[F_{\mathbf{k}_{1}}\right] \alpha\right)}\left(R_{2}\right) \times d^{\left(\left[F_{\mathbf{k}_{2}}\right] \beta\right)}\left(R_{2}\right)\right\} \\
&=\left\{d^{\left(\left[F_{\mathbf{k}_{1}}\right]_{\alpha}\right)}\left(R_{1}\right) \cdot d^{\left(\left[F_{\mathbf{k}_{1}}\right] \alpha\right)}\left(R_{2}\right)\right\} \times\left\{d^{\left(\left[F_{\mathbf{k}_{2}}\right] \beta\right)}\left(R_{1}\right) \cdot d^{\left(\left[F_{\mathbf{k}_{2}}\right] \beta\right)}\left(R_{2}\right)\right\} \\
&=\left\{d^{\left(\left[F_{\mathbf{k}_{1}}\right] \alpha\right)}\left(R_{1} R_{2}\right) \cdot \omega^{\left(\mathbf{k}_{1}\right)}\left(R_{1}, R_{2}\right)\right\} \\
& \times\left\{d^{\left(\left[F_{\mathbf{k}_{2}}\right] \beta\right)}\left(R_{1}, R_{2}\right) \cdot \omega^{\left(\mathbf{k}_{2}\right)}\left(R_{1}, R_{2}\right)\right\} \\
&= d^{\left(\left[F_{\mathbf{k}_{3}}\right]_{\alpha \beta}\right)}\left(R_{1} R_{2}\right) \cdot \omega^{\left(\mathbf{k}_{1}\right)}\left(R_{1}, R_{2}\right) \cdot \omega^{\left(\mathbf{k}_{2}\right)}\left(R_{1}, R_{2}\right) \\
&= d^{\left(\left[F_{\mathbf{k}_{3}}\right] \alpha \beta\right)}\left(R_{1} R_{2}\right) \cdot \omega^{\mathbf{k}_{3}}\left(R_{1}, R_{2}\right)
\end{aligned}
$$

for both (11) and (12) factor systems.

Let the coset representatives $g_{s}^{(j)} \in G_{\mathbf{k}_{j}}$ in the decomposition of $G_{\mathbf{k}_{j}}$ with respect to the translation group $T$

$$
G_{\mathbf{k}_{j}}=\sum_{s} g_{s}^{(j)} T, \quad g_{s}^{(1)} \in G_{\mathbf{k}}
$$

be chosen in the form $g_{s}^{(j)} \equiv\left(R_{s}^{(j)} \mid \mathbf{v}_{s}^{(j)}\right)$, i. e. they are among the representatives $\left(R_{i} \mid \mathbf{v}_{i}\right)$ in the decomposition (1). The element $g_{j} g_{s}^{(1)} g_{j}^{-1} \in G_{\mathbf{k}_{j}}$ with $g_{j}$ being taken from decomposition (4) (see also (6)) may differ from $g_{s}^{(j)}$ by some lattice translation. That is why the notations of the small irreps of the little groups $G_{\mathbf{k}}$ and $G_{\mathbf{k}_{j}}$ (and the projective irreps of the corresponding little co-groups) may be different.

In particular, let $g_{j_{0}} \mathbf{k}=-\mathbf{k}$. The groups $G_{\mathbf{k}}$ and $G_{-\mathbf{k}}$ are composed of the same elements. The whole set of small irreps of the little group $G_{-\mathbf{k}}$ is complex conjugated with respect to the whole set of small irreps of the little group $G_{\mathbf{k}}$, but the notations of the irreps of $G_{\mathbf{k}}$ and of $G_{-\mathbf{k}}$ may differ (see example in Section 3).

Let $Q$ be a group and $H$ be its subgroup $(H \subset Q)$. Let $d^{(\alpha)}$ and $D^{(\beta)}$ be irreps of $H$ and $Q$, respectively. Then the frequency of the irrep $D^{(\beta)}$ of $Q$ in the rep $\left(d^{(\alpha)} \uparrow Q\right)$ induced by the irrep $d^{(\alpha)}$ of $H$ is equal to the frequency of the irrep $d^{(\alpha)}$ of $H$ in the rep $\left(D^{(\beta)} \downarrow H\right)$ subduced by $D^{(\beta)}$ on $H$ (Frobenius reciprocity theorem). This theorem can be applied also to the projective irreps of a group and its subgroup with the same factor system [6].

\section{Procedure of the selection rules' generation using projective representations of point groups}

The stationary states of a system with the symmetry of a space group $G$ are classified according to the irreps of $G$ and their full group-theoretical notation is as follows: $|\mathbf{k}, \gamma, m, \mu\rangle$ where $\mathbf{k}=\mathbf{k}_{1}, \mathbf{k}_{2}, \ldots, \mathbf{k}_{t}$ (star $\left.{ }^{*} \mathbf{k}\right), m$ numbers the basis vectors of the small irrep $\gamma$ of the little group $G_{\mathbf{k}}$, and $\mu$ numbers the independent bases of equivalent reps of $G_{\mathbf{k}}$. 
Let us consider the selection rules for the transitions between the stationary states $\left|\mathbf{k}^{(f)}, \gamma^{(f)}, m^{(f)}, \mu^{(f)}\right\rangle$ and $\left|\mathbf{k}^{(i)}, \gamma^{(i)}, m^{(i)}, \mu^{(i)}\right\rangle$ caused by an operator $P$ $\left(\mathbf{k}^{(p)}, \gamma^{(p)}, m^{(p)}\right)$ transforming according to the irrep $\left(\mathbf{k}^{(p)}, \gamma^{(p)}\right)$ of $G$. If the operator $P$ transforms according to a reducible rep of $G$, one can obtain the selection rules for every of its irreducible components separately.

The transition probability is governed by the value of the matrix element

$$
\left\langle\mathbf{k}^{(f)}, \gamma^{(f)}, m^{(f)}, \mu^{(f)}\left|P\left(\mathbf{k}^{(p)}, \gamma^{(p)}, m^{(p)}\right)\right| \mathbf{k}^{(i)}, \gamma^{(i)}, m^{(i)}, \mu^{(i)}\right\rangle .
$$

The transition is said to be allowed by symmetry if the triple direct (Kronecker) product

$$
\left(\mathbf{k}^{(f)}, \gamma^{(f)}\right)^{*} \times\left(\mathbf{k}^{(p)}, \gamma^{(p)}\right) \times\left(\mathbf{k}^{(i)}, \gamma^{(i)}\right)
$$

contains the identity irrep of $G$. This condition can be rewritten in one of three following forms:

$$
\begin{aligned}
& {\left[\left(\mathbf{k}^{(p)}, \gamma^{(p)}\right) \times\left(\mathbf{k}^{(i)}, \gamma^{(i)}\right)\right] \cap\left(\mathbf{k}^{(f)}, \gamma^{(f)}\right) \neq 0,} \\
& {\left[\left(\mathbf{k}^{(f)}, \gamma^{(f)}\right) \times\left(\mathbf{k}^{(i)}, \gamma^{(i)}\right)^{*}\right] \cap\left(\mathbf{k}^{(p)}, \gamma^{(p)}\right) \neq 0,} \\
& {\left[\left(\mathbf{k}^{(f)}, \gamma^{(f)}\right) \times\left(\mathbf{k}^{(p)}, \gamma^{(p)}\right)^{*}\right] \cap\left(\mathbf{k}^{(i)}, \gamma^{(i)}\right) \neq 0 .}
\end{aligned}
$$

Whatever the form of the selection rules, it is necessary to find the direct product of two (or three (21)) irreps of the space group $G$ (complex conjugate irreps are also irreps of $G)$.

We discuss now the procedure of selection rules' generation using projective irreps of point groups. To illustrate each step of this procedure we have chosen the small irreps of the little group $G_{W}$ in the space group $O_{h}^{7}$ given in Tables [9]. Note that translations $\mathbf{a}_{\mathbf{n}}$ are mapped in Ref. [9] by the factor $\exp \left(i \mathbf{k} \mathbf{a}_{\mathbf{n}}\right)$. According to general definition

$$
\hat{t}_{\mathbf{a}_{\mathbf{n}}} \psi(\mathbf{r}) \equiv \psi\left(\mathbf{r}-\mathbf{a}_{\mathbf{n}}\right)=\exp \left(-i \mathbf{k a}_{\mathbf{n}}\right) \psi(\mathbf{r}),
$$

we choose the translations $\mathbf{a}_{\mathbf{n}}$ to be mapped by the factor $\exp \left(-i \mathbf{k a}_{\mathbf{n}}\right)$. This choice does not affect the notations of Ref. [9] for small irreps in the case when $\mathbf{k}$ is equivalent to $-\mathbf{k}$ or refers the small irreps $\left[G_{\mathbf{k}}\right]_{\gamma}$ of Ref. [9] to the wave vector $-\mathbf{k}$ in other cases (when $\mathbf{k}$ and $-\mathbf{k}$ are different vectors of the same star or belong to different stars).

The star ${ }^{*} W$ consists of six vectors: $W^{(1)}=(1,0,2)$, $W^{(2)}=(1,2,0), \quad W^{(3)}=(2,1,0), \quad W^{(4)}=(0,1,2)$, $W^{(5)}=(0,2,1)$, and $W^{(6)}=(2,0,1)$ (in units of $\pi / a$ along Cartesian axes with $a$ being the lattice constant). The little group $G_{W^{(j)}}$ has two single-valued $\left(\left[G_{W^{(j)}}\right]_{\gamma}, \gamma=1,2\right)$ and five double-valued $\left(\left[G_{W^{(j)}}\right]_{\gamma}, \gamma=3,4,5,6,7\right)$ small irreps $[9,12]$, which are unambiguously related (see Section 2) to the corresponding projective irreps $\left[F_{W^{(j)}}\right]_{j}$ of little co-groups $F_{W^{(j)}}=D_{2 d}^{(j)}$ (see Talbe 1). As the characters (and matrices) of the elements $\left(R \mid \mathbf{v}_{R}\right) \in G_{W^{(j)}}$ and $R \in F_{W^{(j)}}$ are the same, we use the notations $W_{\gamma} \equiv\left[G_{W^{(j)}}\right]_{\gamma} \quad(\gamma=1-7)$ of small irreps of the little groups $G_{W^{(j)}}$ also for the corresponding projective irreps
Table 1. Characters of single- and double-valued projective irreps of the little co-groups $F_{W^{(i)}} \equiv D_{2 d}^{(i)}(i=1-6)$ and singleand double-valued small irreps of the little groups $G_{W^{(i)}}(i=1-6)$ (for six vectors in the star ${ }^{*} W$ : (102), (120), (210), (012), (021) and (201) in the units of $\pi / a$ along Cartesian axes, $a$ being the lattice constant) in the $\mathrm{BZ}$ in crystal with the space group $O_{h}^{7}$, $\varepsilon=\exp (i \pi / 4)$

\begin{tabular}{c|c|c|c|c|c|c|c|c|c}
\hline$D_{2 d}^{(1)}$ & $D_{2 d}^{(2)}$ & $E$ & $S_{4 x}$ & $S_{4 x}^{-1}$ & $C_{2 x}$ & $U_{y z}$ & $U_{y \bar{z}}$ & $\sigma_{y}$ & $\sigma_{z}$ \\
$D_{2 d}^{(3)}$ & $D_{2 d}^{(4)}$ & $E$ & $S_{4 y}$ & $S_{4 y}^{-1}$ & $C_{2 y}$ & $U_{x z}$ & $\bar{U}_{x \bar{z}}$ & $\sigma_{z}$ & $\sigma_{x}$ \\
$D_{2 d}^{(5)}$ & $D_{2 d}^{(6)}$ & $E$ & $S_{4 z}$ & $S_{4 z}^{-1}$ & $C_{2 z}$ & $U_{x y}$ & $\bar{U}_{x \bar{y}}$ & $\sigma_{x}$ & $\sigma_{y}$ \\
\hline$W_{1}$ & $W_{1}^{*}$ & 2 & $\sqrt{2} \varepsilon^{*}$ & $\sqrt{2} \varepsilon$ & 0 & 0 & 0 & 0 & 0 \\
$W_{2}$ & $W_{2}^{*}$ & 2 & $-\sqrt{2} \varepsilon^{*}$ & $-\sqrt{2} \varepsilon$ & 0 & 0 & 0 & 0 & 0 \\
\hline$W_{3}$ & $W_{5}^{*}$ & 1 & $\varepsilon^{*}$ & $-\varepsilon$ & $i$ & 1 & $-i$ & $-\varepsilon^{*}$ & $-\varepsilon$ \\
$W_{4}$ & $W_{6}^{*}$ & 1 & $-\varepsilon^{*}$ & $\varepsilon$ & $i$ & -1 & $i$ & $-\varepsilon^{*}$ & $-\varepsilon$ \\
$W_{5}$ & $W_{3}^{*}$ & 1 & $\varepsilon^{*}$ & $-\varepsilon$ & $i$ & -1 & $i$ & $\varepsilon^{*}$ & $\varepsilon$ \\
$W_{6}$ & $W_{4}^{*}$ & 1 & $-\varepsilon^{*}$ & $\varepsilon$ & $i$ & 1 & $-i$ & $\varepsilon^{*}$ & $\varepsilon$ \\
$W_{7}$ & $W_{7}^{*}$ & 2 & 0 & 0 & $-2 i$ & 0 & 0 & 0 & 0
\end{tabular}

of little co-groups $G_{W^{(j)}}=D_{2 d}^{(j)}$. Since $\mathbf{k}_{W^{(j)}} \neq-\mathbf{k}_{W^{(j)}}$ and $\mathbf{k}_{W^{(2)}} \sim-\mathbf{k}_{W^{(1)}}$ we take the irreps of the little group $G_{W^{(2)}}$ from Ref. [9] as the irreps of the little group $G_{W^{(1)}}$ (see (25) and the remark thereunder). Besides as $\mathbf{k}_{W^{(2)}} \sim-\mathbf{k}_{W^{(1)}}$, the total sets of single- and double-valued irreps of little groups $G_{W^{(1)}}$ and $G_{W^{(2)}}$ are complex conjugate, but the elements of $G_{W^{(2)}}=I G_{W^{(1)}} I^{-1}$ which are isomorphic to the elements of $G_{W^{(1)}}$ according to (6) may differ from the coset representatives in decomposition (4) by some lattice translations. This may change the numbering of the irreps of the little group $G_{W^{(2)}}$ (and projective irreps of the corresponding little co-group $F_{W^{(2)}}$ ) with respect to those of $G_{W^{(1)}}$ (of $F_{W^{(1)}}$, see Table $1 ; W_{3}\left(D_{2 d}^{(2)}\right)=W_{5}^{*}\left(D_{2 d}^{(1)}\right)$, for example).

Taking the form (22) of selection rules, we consider the Kronecker product of the irreps of the space group $G$

$$
[G]_{\gamma^{(p)} \gamma^{(i)}} \equiv[G]_{\gamma^{(p)}}^{\left({ }^{(p)}\right)} \times[G]_{\gamma^{(i)}}^{\left({ }^{*} \mathbf{k}^{(i)}\right)}
$$

whose basis vectors are the products

$$
\begin{gathered}
\left|\mathbf{k}_{n}^{(p)}, \gamma^{(p)}, m^{(p)}\right\rangle \cdot\left|\mathbf{k}_{l}^{(i)}, \gamma^{(i)}, m^{(i)}\right\rangle, \\
\left(n=1, \ldots s^{(p)} ; \quad l=1, \ldots s^{(i)} ;\right. \\
\left.m^{(p)}=1, \ldots t^{(p)} ; \quad m^{(i)}=1, \ldots t^{(i)}\right),
\end{gathered}
$$

where $s^{(p)}, s^{(i)}$ are the numbers of rays in the stars ${ }^{*} \mathbf{k}^{(p)}$, ${ }^{*} \mathbf{k}^{(i)} ; t^{(p)}, t^{(i)}$ are the dimensions of small irreps $\left[G_{\mathbf{k}_{n}^{(p)}}\right]_{\gamma^{(p)}}$ and $\left[G_{\mathbf{k}_{l}^{(i)}}\right]_{\gamma^{(i)}}$ of little groups $G_{\mathbf{k}_{n}^{(p)}}$ and $G_{\mathbf{k}_{l}^{(i)}}$, respectively.

In the case of the $W$ point in the $\mathrm{BZ}$ for the space group $O_{h}^{7}$, the basis (27) of the Kronecker product $(26)$ for $\gamma^{(p)}=\gamma^{(i)}=1 \quad\left({ }^{*} \mathbf{k}^{(p)}={ }^{*} \mathbf{k}^{(i)}={ }^{*} W\right.$; $\left.s^{(p)}=s^{(i)}=6, \quad[G]_{11} \equiv[G]_{1}^{\left(* W^{(p)}\right)} \times[G]_{1}^{\left({ }^{(} W^{(i)}\right)}\right)$ consists of $(2 \times 6) \times(2 \times 6)=144$ vectors. 
Table 2. Types of wave vectors in Kronecker products of $[G]_{i}^{\left({ }^{*} W\right)} \times[G]_{j}^{\left.{ }^{*} W\right)}$

\begin{tabular}{l|l|l|l|l|l|l}
\hline & $W^{(1 i)}$ & $W^{(2 i)}$ & $W^{(3 i)}$ & $W^{(4 i)}$ & $W^{(5 i)}$ & $W^{(6 i)}$ \\
\hline$W^{(1 p)}$ & $X^{(x f)}$ & $\Gamma^{(f)}$ & $\Sigma^{(2 f)}$ & $\Sigma^{(1 f)}$ & $\Sigma^{(6 f)}$ & $\Sigma^{(7 f)}$ \\
$W^{(2 p)}$ & $\Gamma^{(f)}$ & $X^{(x f)}$ & $\Sigma^{(3 f)}$ & $\Sigma^{(4 f)}$ & $\Sigma^{(5 f)}$ & $\Sigma^{(8 f)}$ \\
$W^{(3 p)}$ & $\Sigma^{(2 f)}$ & $\Sigma^{(3 f)}$ & $X^{(y f)}$ & $\Gamma^{(f)}$ & $\Sigma^{(10 f)}$ & $\Sigma^{(9 f)}$ \\
$W^{(4 p)}$ & $\Sigma^{(1 f)}$ & $\Sigma^{(4 f)}$ & $\Gamma^{(f)}$ & $X^{(y f)}$ & $\Sigma^{(11 f)}$ & $\Sigma^{(12 f)}$ \\
$W^{(5 p)}$ & $\Sigma^{(6 f)}$ & $\Sigma^{(5 f)}$ & $\Sigma^{(10 f)}$ & $\Sigma^{(11 f)}$ & $X^{(z f)}$ & $\Gamma^{(f)}$ \\
$W^{(6 p)}$ & $\Sigma^{(7 f)}$ & $\Sigma^{(8 f)}$ & $\Sigma^{(9 f)}$ & $\Sigma^{(12 f)}$ & $\Gamma^{(f)}$ & $X^{(z f)}$
\end{tabular}

Remark. The wave vector stars $\mathrm{X}^{*} X$ and ${ }^{*} \Sigma$ consist of vectors: $X^{(j f)}$ $(i=x, y, z ;(2,0,0),(0,2,0),(0,0,2))$ and $\Sigma^{(j f)}(j=1-12 ;(110),(1 \overline{1} 0)$,

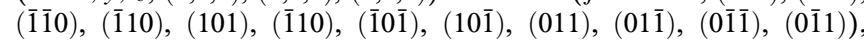
respectively (in the units of $\pi / a$ along Cartesian axes, $a$ being the lattice constant).

Table 3. Types of wave vectors in Kronecker products of $\left({ }^{*} \mathbf{k}_{1}\right) \times\left({ }^{*} \mathbf{k}_{2}\right)\left(\mathbf{k}_{1}, \mathbf{k}_{2}=\Gamma, X, L, W\right)$

\begin{tabular}{c|c|c|c|c|c|c|c}
\hline$\Gamma$ & $X^{(x i)}$ & $X^{(y i)}$ & $X^{(z i)}$ & $L^{(1 i)}$ & $L^{(2 i)}$ & $L^{(3 i)}$ & $L^{(4 i)}$ \\
\hline$X^{(x p)}$ & $\Gamma^{(f)}$ & $X^{(z f)}$ & $X^{(y f)}$ & $L^{(3 f)}$ & $L^{(4 f)}$ & $L^{(1 f)}$ & $L^{(2 f)}$ \\
$L^{(1 p)}$ & $L^{(3 f)}$ & $L^{(4 f)}$ & $L^{(2 f)}$ & $\Gamma^{(f)}$ & $X^{(z f)}$ & $X^{(x f)}$ & $X^{(y f)}$ \\
$W^{(1 p)}$ & $W^{(2 f)}$ & $\Delta^{(2 f)}$ & $\Delta^{(1 f)}$ & $\Sigma^{(12 f)}$ & $\Sigma^{(11 f)}$ & $\Sigma^{(10 f)}$ & $\Sigma^{(9 f)}$ \\
\hline \hline$\Gamma$ & $W^{(1 i)}$ & $W^{(2 i)}$ & $W^{(3 i)}$ & $W^{(4 i)}$ & $W^{(5 i)}$ & $W^{(6 i)}$ \\
\hline$X^{(x p)}$ & $W^{(2 f)}$ & $W^{(1 f)}$ & $\Delta^{(3 f)}$ & $\Delta^{(4 f)}$ & $\Delta^{(6 f)}$ & $\Delta^{(5 f)}$ \\
$L^{(1 p)}$ & $\Sigma^{(12 f)}$ & $\Sigma^{(10 f)}$ & $\Sigma^{(8 f)}$ & $\Sigma^{(6 f)}$ & $\Sigma^{(4 f)}$ & $\Sigma^{(2 f)}$ \\
$W^{(1 p)}$ & $X^{(x f)}$ & $\Gamma^{(f)}$ & $\Sigma^{(2 f)}$ & $\Sigma^{(1 f)}$ & $\Sigma^{(6 f)}$ & $\Sigma^{(7 f)}$ \\
\end{tabular}

Remark. The wave vector stars ${ }^{*} L$ and $\Delta^{*}$ consist of vectors: $L^{(j f)}$ $(j=1-4 ;(1,1,1),(\overline{1}, \overline{1}, 1),(1, \overline{1}, \overline{1}),(\overline{1}, 1, \overline{1}))$ and $\Delta^{(j f)}(j=1-6$; $(1,0,0),(\overline{1}, 0,0),(0,1,0),(0, \overline{1}, 0),(0,0,1),(0,0, \overline{1}))$ (in the units of $\pi / a$ along Cartesian axes, $a$ being the lattice constant).

Decomposing the reducible rep $[G]_{11}$ of $G$, one finds all the irreducible stars ${ }^{*} \mathbf{k}^{(f)}$ contained in the reducible star of $[G]_{11}$ and the small irreps $\left[G_{\mathbf{k}^{(f)}}\right]_{j}$ of little groups $G_{\mathbf{k}^{(f)}}$ contained in the rep $[G]_{11}$. A star of $s^{(p)} s^{(i)}$ wave vectors

$$
\begin{gathered}
\mathbf{k}_{n, l}^{(f)}=\mathbf{k}_{n}^{(p)}+\mathbf{k}_{l}^{(i)}+\mathbf{B}_{n, l} \\
\left(n=1, \ldots s^{(p)}, \quad l=1, \ldots s^{(i)}\right)
\end{gathered}
$$

of basis functions (27) splits into irreducible stars and gives wave vector selection rules (the vector $\mathbf{B}_{n, l}$ is a reciprocal lattice vector which may be zero).

For Kronecker products $[G]_{1}^{\left(* W^{(p)}\right)} \times[G]_{1}^{\left({ }^{*} W^{(i)}\right)}$ of two irreps of $O_{h}^{7}$ at the $W$ point of BZ, the wave vector selection rules (28) give three irreducible stars $(\Gamma, X$ and $\Sigma$, see Table 2). It is easy to see, rewriting the vectors of $W$-star in components of the reciprocal lattice primitive translations. For example, as $W^{(1)}=(1 / 2,3 / 4,1 / 4)$ and $W^{(2)}=(1 / 2,1 / 4,3 / 4)$, one obtains $W^{(1)}+W^{(2)}=(1,1,1)$ $(\sim \Gamma), W^{(1)}+W^{(1)}=(1,3 / 2,1 / 2) \quad\left(\sim X^{(x)}\right)$. Thus, 144 products (27) are partitioned in such a way that $4 \times 6=24$ of them corresponds to $\Gamma, 4 \times 6=24$ to $X$ and $4 \times 4 \times 6=96$ to $\Sigma$ points of the BZ.
The set of wave vectors $\mathbf{k}_{n, l}^{(f)}$ (28) contains all the rays of all the irreducible stars appearing in the Kronecker product (26) and may be arranged in a table similar to Table 2. Rows and columns of this table are numbered by the rays of the irreducible stars ${ }^{*} \mathbf{k}^{(p)}$ and ${ }^{*} \mathbf{k}^{(i)}$ (by $n$ and $l$ ), respectively. The representatives of all the irreducible stars in (26) appear in any row of this table. Indeed, all the rows of the table $\left(n=2, \ldots s^{(p)}\right.$ in (28)) may be obtained from the first one $(n=1)$ by applying the operations $R_{n}^{(p)}$ transforming the wave vector $\mathbf{k}_{1}^{(p)}$ into $\mathbf{k}_{n}^{(p)}$ $\left(n=2, \ldots s^{(p)}\right)$. Under symmetry operations $R_{n}^{(p)}$, the set of wave vectors $\mathbf{k}_{l}^{(i)}$ of the star ${ }^{*} \mathbf{k}^{(i)}$ remains unchanged and the irreducible stars formed by wave vectors $\mathbf{k}_{1, l}^{(f)}$ may change their representatives but can neither disappear nor give raise to new irreducible stars or change the number of each star representatives. The same consideration is valid for the columns. Finally, all the rows (columns) of the whole table contain as many representatives of each irreducible star as the first row (column). Therefore, all the necessary information about wave vector selection rules for the Kronecker product (26) is contained in any row (column) of the corresponding table. The wave vector selection rules for all the symmetry points of BZ of the space group $O_{h}^{7}$ are represented in Table 3. The latter is composed of the first rows of Tables being similar to Table 2 and corresponding to Kronecker products ${ }^{*} \Gamma \times{ }^{*} \Gamma$, ${ }^{*} X \times{ }^{*} X,{ }^{*} L \times{ }^{*} L,{ }^{*} W \times{ }^{*} W,{ }^{*} \Gamma \times{ }^{*} X,{ }^{*} \Gamma \times{ }^{*} L,{ }^{*} \Gamma \times{ }^{*} W$, ${ }^{*} X \times{ }^{*} L,{ }^{*} X \times{ }^{*} W,{ }^{*} L \times{ }^{*} W$.

At the next step of the selection rules' generation one needs to find the irreducible components of the reducible reps for each star satisfying wave vector selection rules.

Let $\mathbf{k}_{m}^{(f)} \equiv \mathbf{k}_{n, l}^{(f)}$ be a wave vector of some irreducible star ( $m$ is fixed). The set of $t^{(p)} t^{(i)}$ basis functions (27) with this wave vector forms the space $\Omega_{1}$ of some projective rep $\left[\tilde{F}_{\mathbf{k}_{m}^{(f)}}\right]_{\mathrm{Kr}} \equiv\left[F_{\mathbf{k}_{n}^{(p)}} \cap F_{\mathbf{k}_{l}^{(i)}}\right]_{\mathrm{Kr}}$ of the little co-group $\tilde{F}_{\mathbf{k}_{m}^{(f)}} \equiv F_{\mathbf{k}_{n}^{(p)}} \cap F_{\mathbf{k}_{l}^{(i)}}$ or small rep $\left[\tilde{G}_{\mathbf{k}_{m}^{(f)}}\right]_{\mathrm{Kr}} \equiv\left[G_{\mathbf{k}_{n}^{(p)}} \cap G_{\mathbf{k}_{l}^{(i)}}\right]_{\mathrm{Kr}}$ of the little group $\tilde{G}_{\mathbf{k}_{m}^{(f)}} \equiv G_{\mathbf{k}_{n}^{(p)}} \cap G_{\mathbf{k}_{l}^{(i)}}$ and

$$
\left[\tilde{F}_{\mathbf{k}_{m}^{(f)}}\right]_{\mathrm{Kr}}=\left[\tilde{G}_{\mathbf{k}_{m}^{(f)}}\right]_{\mathrm{Kr}} \Downarrow \tilde{F}_{\mathbf{k}_{m}^{(f)}} .
$$

The characters of the projective rep $\left[\tilde{F}_{\mathbf{k}_{m}^{(f)}}\right]_{\mathrm{Kr}}$ of the cogroup $\tilde{F}_{\mathbf{k}_{m}^{(f)}}$ in the space $\Omega_{1}$ are the products of the characters of the co-group $F_{\mathbf{k}_{n}^{(p)}}$ and $F_{\mathbf{k}_{l}^{(i)}}$ irreps subduced on the cogroup $\tilde{F}_{\mathbf{k}_{m}^{(f)}}$

$$
\begin{aligned}
& \chi^{\left(\left[\tilde{F}_{\mathbf{k}_{m}^{(f)}}\right] \mathrm{Kr}_{\mathrm{r}}\right)}(R)=\chi^{\left(\left[\tilde{F}_{\mathbf{k}_{n}(p)}\right]_{\gamma(p)}\right)}(R) \chi^{\left(\left[\tilde{F}_{l}^{(i)}\right]_{\gamma^{(i)}}\right)}(R), \\
& R \in \tilde{F}_{\mathbf{k}_{m}}(f) .
\end{aligned}
$$

The multiplication of two projective irreps of the group $\tilde{F}_{\mathbf{k}_{m}^{(f)}}$ with factor systems $\omega^{\left(\mathbf{k}_{n}^{(p)}\right)}$ and $\omega^{\left(\mathbf{k}_{l}^{(i)}\right)}$ gives a projective rep of the same group with the factor system $\omega^{\left(\mathbf{k}_{m}^{(f)}\right)}$ (as $\mathbf{k}_{m}^{(f)} \equiv \mathbf{k}_{n l}^{(f)}=\mathbf{k}_{n}^{(p)}+\mathbf{k}_{l}^{(i)}+\mathbf{B}_{n l}$, see also Section 2). 
The group $\tilde{F}_{\mathbf{k}_{m}^{(f)}}\left(\tilde{G}_{\mathbf{k}_{m}^{(f)}}\right)$ either coincides with the little cogroup $F_{\mathbf{k}_{m}^{(f)}}$ (little group $\left.G_{\mathbf{k}_{m}^{(f)}}\right)$ or is a subgroup of it

$$
\tilde{F}_{\mathbf{k}_{m}^{(f)}} \subseteq F_{\mathbf{k}_{m}^{(f)}} \quad\left(\tilde{G}_{\mathbf{k}_{m}^{(f)}} \subseteq G_{\mathbf{k}_{m}^{(f)}}\right) .
$$

When $\tilde{F}_{\mathbf{k}_{m}^{(f)}}=F_{\mathbf{k}_{m}^{(f)}}$ then the projective rep (30) can be decomposed into the irreps in the usual way, the characters of irreps being taken from Tables of small irreps of little groups (for example [9]). This possibility appears, for instance, when $\mathbf{k}_{n 1}^{(p)}=0$ or $\mathbf{k}_{l 2}^{(i)}=0$.

If $\tilde{F}_{\mathbf{k}_{m}^{(f)}} \subset F_{\mathbf{k}_{m}^{(f)}}$, the co-group $F_{\mathbf{k}_{m}^{(f)}}$ is decomposed into left cosets of $\tilde{F}_{\mathbf{k}_{m}^{(f)}}$

$$
\begin{gathered}
F_{\mathbf{k}_{m}^{(f)}}=\sum_{i=1}^{w} R_{i} \tilde{F}_{\mathbf{k}_{m}^{(f)}}, \quad R_{1}=E, \\
R_{i} \in F_{\mathbf{k}_{m}^{(f)}}, \quad R_{i} \notin \tilde{F}_{\mathbf{k}_{m}^{(f)}} \text { for } i=2, \ldots, w .
\end{gathered}
$$

The operators $R_{i}$ change both wave vectors $\mathbf{k}_{n}^{(p)}$ and $\mathbf{k}_{l}^{(i)}$ but leave their sum unchanged modulo the reciprocal lattice vector. This means that the space $\Omega_{1}$ transforms under the operaitons $R_{i}$ into linearly independent spaces $\Omega_{i}=\hat{R}_{i} \Omega_{1}$, and

$$
\Omega=\sum_{i=1}^{w} \Omega_{i}
$$

being the space of the rep of the group $F_{\mathbf{k}_{m}^{(f)}}$ induced by the rep $\left[\tilde{F}_{\mathbf{k}_{m}^{(f)}}\right]_{\mathrm{Kr}}$ of its subgroup $\tilde{F}_{\mathbf{k}_{m}^{(f)}} \subset F_{\mathbf{k}_{m}^{(f)}}$

$$
\left[F_{\mathbf{k}_{m}^{(f)}}\right]_{\mathrm{Kr}}=\left[\tilde{F}_{\mathbf{k}_{m}^{(f)}}\right]_{\mathrm{Kr}} \uparrow F_{\mathbf{k}_{m}^{(f)}} .
$$

Further, the small rep $\left[G_{\mathbf{k}_{m}^{(f)}}\right]_{\mathrm{Kr}}=\left[F_{\mathbf{k}_{m}^{(f)}}\right]_{\mathrm{Kr}} \Uparrow G_{\mathbf{k}_{m}^{(f)}}$ is contained in the Kronecker product (26) which is the subject of our consideration. The characters $\chi^{\left(\left[F_{\mathbf{k}_{m}}^{(f)}\right]_{\mathrm{rr}}\right)}(g)\left(g \in F_{\mathbf{k}_{m}^{(f)}}\right)$ of this projective induced rep of $F_{\mathbf{k}_{m}^{(f)}}$ (or induced small rep of $\left.G_{\mathbf{k}_{m}^{(f)}}\right)$ can be calculated in the usual way

$$
\chi^{\left(\left[F_{\mathbf{k}_{m}^{(f)}}\right] \mathrm{Kr}\right)}(g)=\sum_{i} \chi_{i}^{(\mathrm{Kr})}(g)
$$

where

$$
\chi_{i}^{(\mathrm{Kr})}(g)= \begin{cases}0, & \text { if } g_{i}^{-1} g g_{i} \notin \tilde{F}_{\mathbf{k}_{m}^{(f)}} ; \\ \chi^{\left(\left[\tilde{F}_{\mathbf{k}_{m}}^{(f)}\right]_{\mathrm{Kr}}\right)}\left(g_{i}^{-1} g g_{i}\right), & \text { if } g_{i}^{-1} g g_{i} \in \tilde{F}_{\mathbf{k}_{m}^{(f)}} .\end{cases}
$$

As the characters of projective irreps of $F_{\mathbf{k}_{m}^{(f)}}$ (of small irreps of $\left.G_{\mathbf{k}_{m}^{(f)}}\right)$ are known (taken from Ref. [9], for example), the projective rep of $\left[F_{\mathbf{k}_{m}^{(f)}}\right]_{\mathrm{Kr}}$ can be decomposed on the irreducible components in the same way as it is made for ordinary reps of point groups.

If the projective irreps $\left[\tilde{F}_{\mathbf{k}_{m}^{(f)}}\right]_{j}$ with the same factor system as the projective irreps of the co-group $F_{\mathbf{k}_{m}^{(f)}}$ are known, there is a more simple procedure of the rep decomposition
Table 4. Characters of the Kronecker product $\left[D_{2 d}^{(1)}\right]_{1} \times\left[D_{2 d}^{(2)}\right]_{1} \equiv \chi$ and the characters of some ordinary

\begin{tabular}{|c|c|c|c|c|c|c|c|}
\hline & $W_{1}$ & $W_{2}$ & $W_{3}$ & $W_{4}$ & $W_{5}$ & $W_{6}$ & $W_{7}$ \\
\hline $\begin{array}{l}W_{1} \\
W_{2}\end{array}$ & $\begin{array}{l}a_{1}, a_{2}, e \\
b_{1}, b_{2}, e\end{array}$ & $\begin{array}{l}b_{1}, b_{2}, e \\
a_{1}, a_{2}, e\end{array}$ & $\begin{array}{l}\bar{e}_{1} \\
\bar{e}_{2}\end{array}$ & $\begin{array}{l}\bar{e}_{2} \\
\bar{\rho}_{1}\end{array}$ & $\begin{array}{l}\bar{e}_{1} \\
\bar{\rho}_{2}\end{array}$ & $\begin{array}{l}\bar{e}_{2} \\
\bar{\rho}_{1}\end{array}$ & $\begin{array}{l}\bar{e}_{1}, \bar{e}_{2} \\
\bar{\rho}_{1} \bar{p}_{2}\end{array}$ \\
\hline & & & & & & & \\
\hline $\begin{array}{l}W_{3} \\
W_{4}\end{array}$ & $\begin{array}{l}\bar{e}_{1} \\
\bar{e}_{2}\end{array}$ & $\begin{array}{l}\bar{e}_{2} \\
\bar{e}_{1}\end{array}$ & $a_{2}$ & $b_{1}$ & $\begin{array}{l}a_{1} \\
b_{2}\end{array}$ & $\begin{array}{l}b_{2} \\
a_{1}\end{array}$ & $\begin{array}{l}e \\
e\end{array}$ \\
\hline $\begin{array}{l}W_{4} \\
W_{5}\end{array}$ & $\bar{e}_{1}$ & $\begin{array}{l}\bar{e}_{2} \\
\text { nat }\end{array}$ & $a_{1}$ & $b_{2}$ & $a_{2}$ & $b_{1}$ & $e$ \\
\hline$W_{6}$ & $\bar{e}_{2}$ & $\bar{e}_{1}$ & $b_{2}$ & $a_{1}$ & $b_{1}$ & $a_{2}$ & \\
\hline$W_{7}$ & $\bar{e}_{1}, \bar{e}_{2}$ & $\bar{e}_{1}, \bar{e}_{2}$ & $e$ & $e$ & $e$ & $e$ & $a_{1}, a_{2}, b_{1}, b_{2}$ \\
\hline \multicolumn{2}{|c|}{$\left[O_{h}\right]_{i}\left(\Uparrow O_{h}^{7}\right)$} & $\Gamma_{1}^{+} \quad \Gamma_{2}^{+}$ & $\Gamma_{3}^{+}$ & $\Gamma_{4}^{+}$ & $\Gamma_{5}^{+}$ & $\Gamma_{6}^{+}$ & $\Gamma_{7}^{+}$ \\
\hline \multicolumn{2}{|c|}{$\left[O_{h}\right]_{i} \downarrow D_{2 d}$} & $\begin{array}{lll}a_{1} & b_{2} & a\end{array}$ & $a_{1}, b_{2}$ & $a_{2}, e$ & $b_{1}, e$ & $\bar{e}_{2}$ & $\bar{e}_{1} \quad \bar{e}_{1}, \bar{e}_{2}$ \\
\hline \multicolumn{2}{|c|}{$\left[O_{h}\right]_{i}\left(\Uparrow O_{h}^{7}\right)$} & $\Gamma_{1}^{-} \quad \Gamma_{2}^{-}$ & $\Gamma_{3}^{-}$ & $\Gamma_{4}^{-}$ & $\Gamma_{5}^{-}$ & $\Gamma_{6}^{-}$ & $\Gamma_{7}^{-}$ \\
\hline \multicolumn{2}{|c|}{$\left[O_{h}\right]_{i} \downarrow D_{2 d}$} & $b_{1} \quad a_{2} \quad a$ & $a_{2}, b_{1}$ & $b_{2}, e$ & $a_{1}, e$ & $\bar{e}_{1}$ & $\bar{e}_{2} \quad \bar{e}_{1}, \bar{e}_{2}$ \\
\hline
\end{tabular}
irreps of the point group $D_{2 d}^{(1)}$

\begin{tabular}{c|c|c|c|c|r|r|r|r}
\hline$D_{2 d}^{(1)}$ & $E$ & $S_{4 x}$ & $S_{4 x}^{-1}$ & $C_{2 x}$ & $U_{y z}$ & $U_{y \bar{z}}$ & $\sigma_{y}$ & $\sigma_{z}$ \\
\hline$\chi$ & 4 & 2 & 2 & 0 & 0 & 0 & 0 & 0 \\
\hline$a_{1}$ & 1 & 1 & 1 & 1 & 1 & 1 & 1 & 1 \\
$a_{2}$ & 1 & 1 & 1 & 1 & -1 & -1 & -1 & -1 \\
$e$ & 2 & 0 & 0 & -2 & 0 & 0 & 0 & 0
\end{tabular}

Table 5. Kronecker products $\left[D_{2 d}^{(1)}\right]_{i} \times\left[D_{2 d}^{(2)}\right]_{j}$ of projective singleand double-valued irreps of the little co-group $D_{2 d}$ in terms of ordinary irreps of the point group $D_{2 d}$ and subduction of ordinary irreps of $O_{h}$ point group onto the point group $D_{2 d}^{(1)}$

based on the Frobenius reciprocity theorem (see Section 2). Such possibility arises in the two following cases:

a) when the rep $\left[\tilde{F}_{\mathbf{k}_{m}^{(f)}}\right]_{\mathrm{Kr}}$ is irreducible itself, i.e. its characters satisfy the condition

$$
\sum_{g \in \tilde{F}}\left|\chi^{([\tilde{F}])}(g)\right|^{2}=n_{\tilde{F}}
$$

where $n_{\tilde{F}}$ is the order of $\tilde{F}$ (see Section 3.2);

b) when the subduction of the irreps of the co-group $F_{\mathbf{k}_{m}^{(f)}}$ onto the group $\tilde{F}_{\mathbf{k}_{m}^{(f)}}$ gives directly the irreps of $\tilde{F}_{\mathbf{k}_{m}^{(f)}}$.

Besides, the irreps of $\tilde{F}_{\mathbf{k}_{m}^{(f)}}$ can be taken from Ref. [6] where the characters of the standard form for all the projective irreps with all the possible factor systems for all the crystallographic point groups are given.

In our example of the $[G]_{1}^{\left.*^{*} W^{(p)}\right)} \times[G]_{1}^{\left(* W^{(i)}\right)}$ Kronecker product, the following intersections of point groups are considered: $\tilde{F}_{\Gamma} \equiv F_{W^{(1)}} \cap F_{W^{(2)}}\left(D_{2 d}^{(1)} \cap D_{2 d}^{(2)}=D_{2 d}^{(1)}\right)$ for r-component, $\tilde{F}_{X^{(x)}} \equiv F_{W^{(1)}} \cap F_{W^{(1)}}\left(D_{2 d}^{(1)} \cap D_{2 d}^{(1)}=D_{2 d}^{(x)}\right)$ for $X$-component, and $\tilde{F}_{\Sigma} \equiv F_{W^{(1)}} \cap F_{W^{(4)}}\left(D_{2 d}^{(1)} \cap D_{2 d}^{(4)}=C_{s}\right)$ for $\Sigma$-component (see Table 2 or 3 ). 
Table 6. $\Gamma$ states in the Kronecker products $[G]_{i}^{(* W)} \times[G]_{j}^{(* W)}$

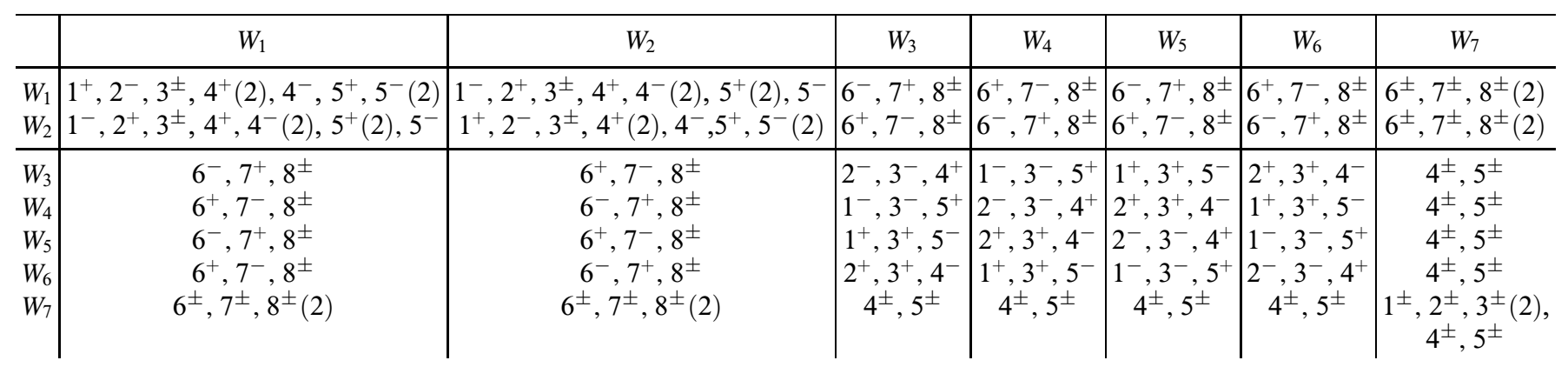

Re mark. Numbers $(m)$ in parentheses mean that the preceding irrep enters $m$ times in the product.

3.1. $\Gamma$ states in the Kronecker products $[G]_{i}^{\left({ }^{*} W\right)} \times[G]_{j}^{\left({ }^{*} W\right)}$. In this case, the irreps of $\tilde{F}_{\mathbf{k}_{m}^{(f)}}=\tilde{F}_{\Gamma}$ are ordinary irreps of the point group $D_{2 d}^{(1)}$. The characters of the Kronecker product of the projective irreps $\left[F_{W^{(1)}}\right]_{1} \times\left[F_{W^{(2)}}\right]_{1} \equiv\left[D_{2 d}^{(1)}\right]_{1} \times\left[D_{2 d}^{(2)}\right]_{1}$ obtained from Table 1 are given in Table 4 with the characters of those ordinary irreps of the little co-group $D_{2 d}^{(1)}$ which appear in the decomposition

$$
\left[D_{2 d}^{(1)}\right]_{1} \times\left[D_{2 d}^{(2)}\right]_{1}=a_{1}+a_{2}+e .
$$

This rep of $D_{2 d}^{(1)}$ induces into $F_{\Gamma}=O_{h}$ the rep $\Gamma_{1}^{+} \Gamma_{2}^{-} \Gamma_{3}^{ \pm} \Gamma_{4}^{ \pm} \Gamma_{5}^{ \pm} \Gamma_{4}^{+} \Gamma_{5}^{-}$. This decomposition of the induced rep is obtained using the Frobenius reciprocity theorem (see Table 5).

Table 7. Characters of single- and double-valued projective irreps of the little co-group $D_{2 d}^{(x)}$ with the factor system corresponding to the little co-group $D_{4 h}^{(x)}$

\begin{tabular}{c|c|c|c|c|c|c|c|c}
\hline$D_{2 d}$ & $E$ & $S_{4 x}$ & $S_{4 x}^{-1}$ & $C_{2 x}$ & $U_{y z}$ & $U_{y \bar{z}}$ & $\sigma_{y}$ & $\sigma_{z}$ \\
\hline$\left[D_{2 d}^{(x)}\right]_{1} \equiv 1$ & 1 & $-i$ & $i$ & -1 & 1 & -1 & $-i$ & $i$ \\
{$\left[D_{2 d}^{(x)}\right]_{2} \equiv 2$} & 1 & $i$ & $-i$ & -1 & -1 & 1 & $-i$ & $i$ \\
{$\left[D_{3 d}^{(x)}\right]_{3} \equiv 3$} & 1 & $-i$ & $i$ & -1 & -1 & 1 & $i$ & $-i$ \\
{$\left[D_{2 d}^{(x)}\right]_{4} \equiv 4$} & 1 & $i$ & $-i$ & -1 & 1 & -1 & $i$ & $-i$ \\
{$\left[D_{2 d}^{(x)}\right]_{5} \equiv 5$} & 2 & 0 & 0 & 2 & 0 & 0 & 0 & 0 \\
\hline$\left[D_{2 d}^{(x)}\right]_{6} \equiv 6$ & 2 & $\sqrt{2} i$ & $\sqrt{2} i$ & 0 & 0 & 0 & 0 & 0 \\
{$\left[D_{2 d}^{(x)}\right]_{7} \equiv 7$} & 2 & $-\sqrt{2} i$ & $-\sqrt{2} i$ & 0 & 0 & 0 & 0 & 0
\end{tabular}

The same procedure may be used for all possible Kronecker products $\left[D_{2 d}^{(1)}\right]_{i} \times\left[D_{2 d}^{(2)}\right]_{j}(i, j=1-7)$ both for single- and double-valued irreps. As a result, one obtains Table 5 where the subduction of single- and double-valued ordinary irreps of $O_{h}$ on $D_{2 d}^{(1)}$ are also given. For example, the direct product $[G]_{3}^{\left({ }^{*} W\right)} \times[G]_{4}^{(* W)}$ has the $\Gamma$-component $\Gamma_{1}^{-} \Gamma_{3}^{-} \Gamma_{5}^{+}\left(\right.$rep $\left[O_{h}\right]_{\mathrm{Kr}}$ is induced from the ordinary irrep $b_{1}$ of $D_{2 d}^{(1)}$ ). After induction (15), one obtains Table 6 that gives directly the $\Gamma$-components of the Kronecker products involved.
3.2. $X$ states in the Kronecker products $[G]_{i}^{\left({ }^{*} W\right)} \times[G]_{j}^{\left({ }^{*} W\right)}$. The irreps of $\tilde{F}_{X^{(x)}} \subset D_{4 h}^{(x)}$ may be obtained directly from Table 1 by multiplying $\left[D_{2 d}^{(1)}\right]_{3}$ on $\left[D_{2 d}^{(1)}\right]_{3-7}$ (for the single-valued projective irreps $\left[D_{2 d}^{(x)}\right]_{1-5}$ ) and $\left[D_{2 d}^{(1)}\right]_{1-2}$ (for the double-valued projective trreps $\left[D_{2 d}^{(x)}\right]_{6-7}$ ), respectively (see Table 7).

As seen from Table 2 for ${ }^{*} \mathbf{k}^{(p)}={ }^{*} \mathbf{k}^{(i)}={ }^{*} W$, $\gamma^{(p)}=\gamma^{(i)}=1$, the space of eight functions (27) with $n=l=1,2, t^{(p)}=t^{(i)}=2$ transforms according to some small rep of the little co-group $D_{4 h}^{(x)}$. Four function (27) with $n=l=1, t^{(p)}=t^{(i)}=2$ transform according to the projective rep

$$
\alpha \equiv\left(D_{2 d}^{(1)}\right)_{1} \times\left(D_{2 d}^{(1)}\right)_{1}=\left(D_{2 d}^{(x)}\right)_{1}+\left(D_{2 d}^{(x)}\right)_{3}+\left(D_{2 d}^{(x)}\right)_{5}
$$

of the point group $D_{2 d}^{(x)}=D_{2 d}^{(1)}$ with the factor system corresponding to the little co-group $D_{4 h}^{(x)}$. The functions (27) with $n=l=1$ and $n=l=2$ can be considered as basis functions of the projective rep of the little co-group $D_{4 h}^{(x)}$ induced by the rep $\alpha$ (39) of $D_{2 d}^{(x)}$ with the factor system corresponding to the little group $D_{4 h}^{(x)}$. This induction can be made using the Frobenius theorem.

Table 8. Kronecker products $\left[D_{2 d}^{(1)}\right]_{i} \times\left[D_{2 d}^{(1)}\right]_{j}$ in terms of the projective irreps of $D_{2 d}^{(x)}$ (with the factor system of the little cogroup $D_{4 h}^{(x)}$, Table 7) and subduction of the projective irreps of the little co-group $D_{4 h}^{(x)}$ onto the point group $D_{2 d}^{(x)}$

\begin{tabular}{c|c|c|c|c|c|c|c}
\hline & $W_{1}$ & $W_{2}$ & $W_{3}$ & $W_{4}$ & $W_{5}$ & $W_{6}$ & $W_{7}$ \\
\hline$W_{1}$ & $1,3,5$ & $2,4,5$ & 6 & 7 & 6 & 7 & 6,7 \\
$W_{2}$ & $2,4,5$ & $1,3,5$ & 7 & 6 & 7 & 6 & 6,7 \\
\hline$W_{3}$ & 6 & 7 & 1 & 2 & 3 & 4 & 5 \\
$W_{4}$ & 7 & 6 & 2 & 1 & 4 & 3 & 5 \\
$W_{5}$ & 6 & 7 & 3 & 4 & 1 & 2 & 5 \\
$W_{6}$ & 7 & 6 & 4 & 3 & 2 & 1 & 5 \\
$W_{7}$ & 6,7 & 6,7 & 5 & 5 & 5 & 5 & $1,2,3,4$ \\
\hline
\end{tabular}


Table 9. $X$ states in the Kronecker products $[G]_{i}^{\left({ }^{*} W\right)} \times[G]_{j}^{\left({ }^{*} W\right)}$

\begin{tabular}{c|c|c|c|c|c|c|c}
\hline & $W_{1}$ & $W_{2}$ & $W_{3}$ & $W_{4}$ & $W_{5}$ & $W_{6}$ & $W_{7}$ \\
\hline$W_{1}$ & $1,2,3,4$ & $1,2,3,4$ & 5 & 5 & 5 & 5 & $5(2)$ \\
$W_{2}$ & $1,2,3,4$ & $1,2,3,4$ & 5 & 5 & 5 & 5 & $5(2)$ \\
\hline$W_{3}$ & 5 & 5 & 4 & 3 & 3 & 4 & 1,2 \\
$W_{4}$ & 5 & 5 & 3 & 4 & 4 & 3 & 1,2 \\
$W_{5}$ & 5 & 5 & 3 & 4 & 4 & 3 & 1,2 \\
$W_{6}$ & 5 & 5 & 4 & 3 & 3 & 4 & 1,2 \\
$W_{7}$ & $5(2)$ & $5(2)$ & 1,2 & 1,2 & 1,2 & 1,2 & $3(2), 4(2)$
\end{tabular}

Remark. Numbers $(m)$ in parentheses mean that the preceding irrep enters $m$ times in the product.

At the same time, they are the basis functions of the small rep of the little group $G_{X^{(x)}}$ contained in the basis of the Kronecker product (26) and which, due to the relation

$$
[G]_{W W}^{\left({ }^{*} X\right)}=\left(\left(\alpha \uparrow F_{X^{(x)}}\right) \Uparrow G_{X^{(x)}}\right) \uparrow G,
$$

determines all the ${ }^{*} X$-components in the Kronecker product (26). For example, the single-valued irreps $\left[G_{X}\right]_{i}$ $i=1,2,3,4$ are contained in (26) (see Table 8 where all the Kronecker products $\left[D_{2 d}^{(1)}\right]_{i} \times\left[D_{2 d}^{(1)}\right]_{j}$ and the subduction of single- and double-valued projective irreps of $D_{4 h}^{(x)}$ on $D_{2 d}^{(x)}$ are given). After induction (15), one obtains Table 9 that gives directly $X$-components of the Kronecker products involved.

\section{Selection rules for electrical dipole transitions}

The symmetry of the dipole operator is the vector rep of $O_{h}^{7}: \quad \Gamma_{v}=\left[G_{\Gamma}\right]_{4-}=\Gamma_{4}^{-}$. Since the vector $\mathbf{k}^{(p)}=0$, $\mathbf{k}_{m}^{(f)}=\mathbf{k}_{l}^{(i)}$ (the so called direct transitions $\Gamma \leftrightarrow \Gamma, X \leftrightarrow X$, $L \leftrightarrow L, W \leftrightarrow W$ etc are only allowed). The symmetry of allowed final states for $W \leftrightarrow W$ transitions is pointed out in Table 5 by the entries of the columns containing $b_{2}$ and $e\left(\Gamma_{4}^{-} \downarrow D_{2 d}=b_{2}+e\right)$ in the row corresponding to the symmetry of the initial state. For example, the direct transition is allowed from the initial state of symmetry $W_{3}$ to the final states of symmetry $W_{6}$ and $W_{7}$.

In the case of phonon-assisted electric dipole transitions, these selection rules have to be supplemented with the selection rules where the operator has the symmetry of the phonon participating in the transition. In silicon crystal $\mathrm{Si}$ atoms occupy the site $a$ of symmetry $T_{d}$. The symmetries of phonons in this crystal are given by the rep of the space group $G=O_{h}^{7}$ induced (indrep) by the vector rep $t_{2}$ of the site symmetry group $T_{d}[8,11]$. The short symbol of this indrep is

$$
\Gamma\left(4^{-}, 5^{+}\right), X(1,3,4), L\left(1^{+}, 2^{-}, 3^{+}, 3^{-}\right), W(1,2,2) .
$$

It gives symmetry of phonons at the symmetry points of the BZ. For example, the electric dipole transitions are allowed from the initial electronic $W_{3}$ state to the intermediate $W_{6}$ and $W_{7}$ states (when spin-orbit interaction is taken into account, see Table 5). From these states, with assistance of the phonons of symmetry $W_{1}$, the transitions are allowed in the final $\Gamma$ and $X$ states of symmetry $\Gamma_{6}^{+}, \Gamma_{7}^{-}, \Gamma_{8}^{ \pm}, X_{5}$ and $\Gamma_{6}^{ \pm}, \Gamma_{7}^{ \pm}, 2 \Gamma_{8}^{ \pm}, 2 X_{5}$ (see Table 5,8).

\section{Conclusions}

Our approach to the selection rules in crystals is based on the projective irreps of point groups and consists of three steps.

1) At first, one finds the wave vector selection rules. The results may be given in the form of tables where the rows and columns are numbered by wave vectors of the direct product factors. Any row (column) of this table contains the representatives of all the irreducible stars of the Kronecker product.

2) Next, it is sufficient to fix one row (the first wave vector) of this table and then consider only columns (the second wave vector) giving on the intersection with the chosen row the wave vectors of different irreducible stars. Each wave vector is related to some little co-group. Two co-groups correspond to the two factors in the Kronecker product and the third - to the resulting one. The intersection of two former co-groups is also a subgroup of the resulting co-group (the corresponding wave vectors satisfy the wave vector selection rule). The Kronecker product of the projective irreps of these co-groups taken on elements of their intersection is a small projective rep with the needed factor system of the resulting co-group and can be decomposed on the irreducible components, if the projective irreps of the latter are known.

3) At last, the induction procedure from the projective rep of the two initial co-groups intersection to the resulting little co-groups is realized in order to find the definitive selection rules for allowed transitions (subduction coefficients of Kronecker products). The Frobenius reciprocity theorem may be used at this stage, if the projective rep of the cogroups intersection is decomposed into the irreducible ones.

The suggested approach seems to be the most easy-touse, if comparing to the traditional subgroup [1-4] and full grup [5] methods. It does not depend either on the choice of the coordinate system origin and of the k-star vectors in the description of space groups and their small irreps, or on the form of presentation of irreps of space groups (small irreps of little groups $[9,10]$ or $p$-equivalent projective irreps of little co-groups [11]). Our approach may be easily supplemented to the computer program generating the irreps of space groups given at Bilbao Crystallographic Server $[12,13]$. 


\section{References}

[1] R.J. Elliott, R.J. Loudon. J. Phys. Chem. Solids 15, 146 (1960).

[2] M. Lax, J. Hopfield. Phys. Rev 124, 115 (1961).

[3] C.J. Bradley, A.P. Cracknell. The mathematical theory of symmetry in solids. Clarendon, Oxford (1972).

[4] A.P. Cracknell, B.L. Davis, S.C. Miller, W.F. Love. Kronecker products Tables. Vol. 1-4. Plenum, N.Y. (1979).

[5] J.L. Birman. Theory of crystal space groups and infra-red and Raman lattice processes of insulating crystals. Handbuch der physik. Band XXV/2b. Springer-Verlag, Berlin (1974).

[6] G.L. Bir, G.E. Pikus. Symmetry and strain-induced effects in semiconductors. John Wiley \& Sons, N.Y. (1974).

[7] S.L. Altmann. Induced Representations in Cyrstals and Molecules. London (1977).

[8] R.A. Evarestov, V.P. Smirnov. Site Symmetry in Crystals: Theory and Applications. Vol. 108. of Springer Series in Solid State Sciences, 2nd ed. Berlin (1997).

[9] S.C. Miller, W.F. Love. Tables of Irreducible Representations of Space Groups and Co-Representations of Magnetic Space Groups. Pruett, Boulder (1967).

[10] The irreducible representations of space groups / Ed. by J. Zak. Benjanin, Elmsford. N.Y. (1969).

[11] O.V. Kovalev. Representations of the crystallographic space groups: irreducible repersentations, induced representations and corepresentations 2nd ed. Gordon and Breach, Philadelphia, PA (1993).

[12] E. Kroumova, C. Capillas, A. Kirov, M.I. Aroyo, H. Wondratschek, S. Ivantchev, J.M. Madariaga, J.M. Perez-Mato. Bilbao Crystallographic Server, www.cryst.ehu.es.

[13] S. Ivantchev, E. Kroumova, J.M. Madariaga, J.M. Perez-Mato, M.I. Aroyo. J. Appl. Cryst. 33, 1190 (2000). 